\title{
L'image à la lettre, sous la direction de Nathalie Preiss et Joëlle Raineau
}

\section{Luigi Luison}

\section{(2) OpenEdition \\ 1 Journals}

\section{Edizione digitale}

URL: https://journals.openedition.org/studifrancesi/26992

DOI: 10.4000/studifrancesi.26992

ISSN: 2421-5856

\section{Editore}

Rosenberg \& Sellier

\section{Edizione cartacea}

Data di pubblicazione: 1 avril 2007

Paginazione: 229-230

ISSN: 0039-2944

\section{Notizia bibliografica digitale}

Luigi Luison, «L'image à la lettre, sous la direction de Nathalie Preiss et Joëlle Raineau», Studi Francesi [Online], 151 (LI | I) | 2007, online dal 30 novembre 2015, consultato il 23 novembre 2021. URL: http:// journals.openedition.org/studifrancesi/26992 ; DOI: https://doi.org/10.4000/studifrancesi.26992

Questo documento è stato generato automaticamente il 23 novembre 2021.

\section{(c) (i) (9)}

Studi Francesi è distribuita con Licenza Creative Commons Attribuzione - Non commerciale - Non opere derivate 4.0 Internazionale. 


\title{
L'image à la lettre, sous la direction de Nathalie Preiss et Joëlle Raineau
}

\author{
Luigi Luison
}

\section{NOTIZIA}

AA. VV., L'image à la lettre, sous la direction de Nathalie PREISS et Joëlle RAINEAU, Paris, Éditions des Cendres \& Paris-Musées, 2005, pp. 314.

1 I saggi raccolti in questo volume esprimono le diverse teorie sul rapporto tra il testo e l'immagine. Si può parlare di leggere un'immagine e vedere un testo? I punti di unione o di rottura tra questi due differenti sistemi espressivi sono i fondamenti che spingono gli studiosi, e in particolare quelli che hanno reso possibile la realizzazione di questo volume, alla formulazione di teorie specifiche. Il difficile equilibrio tra rendere leggibile il visibile e visibile il leggibile rappresenta ancora oggi una problematica piuttosto articolata. La Rhétorique de l'image di Roland Barthes (1964) e La struttura assente di Umberto Eco (1968) sono i primi due approcci a questa problematica, che hanno creato dei modelli di riflessione sul legame tra il testo e l'immagine. I presupposti formulati dai due studiosi hanno fornito materia al proliferare di nuove ricerche.

2 L'obiettivo principale auspicato dagli autori dei saggi qui contenuti, è di esporre le attuali teorie provenienti dalle diverse discipline: lingua e letteratura francese, letteratura comparata, filosofia, storia dell'arte, archeologia. I vari studi, che implicano una attenta analisi delle immagini fisse e non in movimento, interessano le civiltà arcaiche, l'iconografia antica e contemporanea, le pitture rupestri, i geroglifici, quadri ed illustrazioni, nonché documenti fotografici. Il confronto tra le varie discipline risulta utile perché facilita la conoscenza ed apre nuove teorie scientifiche grazie ad un approccio storiografico, ermeneutico e critico.

3 Il modo in cui il senso raggiunge il testo e l'immagine e le modalità con cui lo si individua è il filo conduttore della sezione "Lire et interpréter". I tre saggi che ne fanno parte, nel sottolineare la necessità di tenere conto sia dell'intenzione dell'autore, che è 
creatore ed interprete, che di quella del lettore, delineano un'ermeneutica che unisce storiografia, semiologia, poetica e pragmatica, ma che rivela anche una complessità di lettura dell'immagine ed una feconda riflessione teorica su questi concetti.

Il saggio di Bernard vouilloux (Textes et images en regard, pp. 21-57) espone il concetto secondo cui il confronto tra una data immagine ed il testo che ne condivide lo stesso referente, rende possibile l'individuazione di inevitabili distorsioni tra ciò che l'immagine illustra e ciò che il testo descrive. L'elaborazione di tale concetto si concretizza alla luce di quattro aspetti fondamentali che l'A. prende in considerazione e che riguardano l'immagine fissa: 1) funzionerebbe sempre grazie ai particolari; 2) ridurrebbe i tempi verbali ad un "presente continuo"; 3) ignorerebbe la negazione sintattica e quindi sarebbe sempre affermativa; 4) non disporrebbe di strumenti che permettano di contrassegnare alcune relazioni logiche come la casualità e la contraddizione.

5 Il filosofo Christophe GENIN (La lettre et l'image à la même enseigne, pp. 59-81), riflettendo sull'identità dell'immagine, lega l'analisi di ogni tipo di immagine a quella dei diversi tipi di testi ai quali le immagini stesse rimandano. In particolare, l'A. sviluppa un discorso estetico su un'ermeneutica dell'immagine che si colloca tra altre due teorie contigue: la storia dell'arte e la filosofia. Attraverso l'uso di un metodo analitico, comparativo e induttivo, l'A. sostiene che non sia corretto parlare di generalizzazione, poiché la polisemia stessa delle immagini e dei testi deve incitare alla prudenza. Nell'analisi di un'immagine, di un testo e delle loro relazioni è opportuno, secondo l'A., non tralasciare l'aspetto imperativo che taluni ambiti disciplinari impongono, e tenere conto dell'inscrizione di una certa singolarità nel reale.

6 Il saggio di Annie DUPRAT (Jacques-Marie Boyer de Nîmes et l'image polémique, pp. 83-106), imperniato sull'analisi della caricatura come immagine-propaganda, prende in esame i concetti pedagogici di Boyer de Nîmes, il quale, attraverso la produzione di 38 incisioni, ha approssimativamente mantenuto un equilibrio tra testo e immagine, nonostante le sue descrizioni di immagini mostrino un eccesso di precisione, mentre i suoi commenti siano talvolta molto lontani dai documenti presi in esame.

7 La seconda parte del volume, "Modèles croisés, modèles établis", contiene due saggi che riguardano il XIX secolo, importante per la proliferazione di riproduzioni di incisioni su legno e acciaio, e per i nuovi processi di riproduzione come la litografia e la fotografia. Con queste novità rivoluzionarie si assiste ad un rovesciamento dell'ordine tra il testo $\mathrm{e}$ l'immagine, tra il modello e la copia. È l'illustrazione, sostiene l'A., che genera un nuovo testo e gli impone la sua logica; è la copia che crea il tipo divenuto archetipo e poi stereotipo.

8 Alain-Marie BASSY (Images en abyme. Prudhomme-Monnier-Prudhomme, pp. 109-135) basa la sua analisi sulle illustrazioni di Henry Monnier. Inventore di una forma di illustrazione quale la scena figurata, che non si caratterizza in relazione ad un testo ma ad una realtà costruita, Monnier mette in atto una scrittura ed un'immagine che si riflettono l'un l'altra, creando il grande gioco di illusione che è l'effetto specchio. "Come le parole di spirito hanno un doppio senso per coloro che le sanno capire, così", rivela Monnier, "le rappresentazioni hanno una doppia visuale per coloro che le sanno osservare".

9 Il lavoro di Évanghélia STEAD (Le voyage des images du Faust I de Goethe. Lecture, réception et iconographie extensive et intensive au XIX ${ }^{\text {ème }}$ siècle, pp. 137-168) vuole attirare l'attenzione su un tipo di analisi che legherebbe la mythocritique all'iconografia faustiana, 
specialmente alle incisioni ed alle litografie. L'A. mette l'accento sul ruolo dell'immagine nella formazione dell'immaginario collettivo, grazie alla costituzione di una iconografia che si potrebbe chiamare estensiva.

10 La terza parte del volume, "L'image à l'épreuve de l'histoire de l'art: l'image captive?", raccoglie alcuni lavori che coinvolgono l'ambito disciplinare della storia dell'arte. Philippe KAENEL (Iconologie et illustration: autour d'Erwin Panofsky, pp. 171-199) apre il dibattito sullo statuto dell' "illustrazione » e ne prende in esame le due visioni antagoniste (il processo riflessivo e dinamico), che entrano in gioco negli scritti di Erwin Panofsky. Il primo processo constata la sottomissione delle immagini in relazione ai testi, mentre il secondo lavora sulle interazioni tra modi e spazi di rappresentazione e le relazioni dialettiche tra lo scritto ed il visibile. Processo, questo, che denota un equilibrio tra testo e immagine. Questo equilibrio, però, risulta piuttosto instabile secondo la visione di Michel меLот (Illustrer l'histoire de l'art, pp. 201-224), che presenta uno studio sull'opera di Malraux, Écrits sur l'art. Partendo dalla tesi secondo cui gli scritti di Malraux avrebbero introdotto una rottura nel discorso sull'arte, l'A. estende poi la sua analisi alle illustrazioni presenti negli scritti di Malraux, per dimostrare che le immagini da lui scelte operano un cambiamento radicale all'interno dello statuto dell'illustrazione. Con Malraux è l'immagine fotografica che costituisce l'opera d'arte come tale e che crea la propria storia dell'arte, portando in secondo piano il testo dello storico.

11 La quarta ed ultima parte, intitolata "L'image telle qu'en elle-même", prende in esame la possibilità che l'immagine possa produrre dei concetti senza l'ausilio del linguaggio verbale. In tale contesto, Yves GAGNeUX (Langage et image, pp. 227- 252) sviluppa un'idea concepita dal Prof. Jean Gagnepain dell'Université Rennes II, riguardante la capacità umana a creare, definita artistique, e ne analizza le incidenze sul rapporto tra immagine e linguaggio.

Infine, prendendo come spunto i principi dell'ideogramma, Anne-Marie CHRISTIN (Pour une sémiotique visuelle: les leçons de l'idéogramme, pp. 253-273) esamina la pittura di Manet, la quale, secondo l'A., è una pittura da leggere piuttosto che da vedere. Il tipo di lettura a cui si riferisce l'A. non consiste nell'individuare, nei dipinti, simboli o allegorie, bensì una sorta di mascheramento ed una parvenza teatrale. Questa interpretazione mette in evidenza, nella tradizione pittorica, un gioco complesso con il reale. Come l'ideogramma, le opere del pittore articolano lo spazio reale e lo spazio fittizio, lungi da ogni volontà di rappresentazione.

13 Il volume, che non vuole proporre una nuova teoria dei rapporti tra testo e immagine, ma mettere a confronto diversi punti di vista sull'argomento, si chiude con una ricca bibliografia selettiva. 